\title{
Realistic expectations of laboratory testing
}

\author{
JOHN J. FERNANDES, DO
}

The ordering, reviewing, and interpreting of laboratory tests require recognition and familiarity with the concepts of normal values, sensitivity, specificity, predictive values, and prevalence. The significance of these test properties in clinical decision-making is explained.

(Key words: Laboratory tests, sensitivity, specificity, predictive value, prevalence)

The diagnostic usefulness of a laboratory test rests with that test's ability to exclude or confirm a diagnostic hypothesis, to monitor the course of disease, or to detect clinically unsuspected disease. Tests so used can be technically reliable; that is, they are accurate and precise analytically, but of little clinical value if improperly used.

Clinical interpretation requires an understanding of the diagnostic sensitivity, specificity, and predictive value of a laboratory test. These properties, when appropriately applied with the concept of prevalence, will guide clinical screening or profiling, enhance pattern recognition in exclusion and confirmation testing, and confirm an hypothesis.

\section{Normal values}

The normal range of laboratory results as cur-

From the Department of Pathology and Laboratory Medicine, Philadelphia College of Osteopathic Medicine, Philadelphia, $\mathrm{Pa}$, where Dr Fernandes was formerly chairman.

Reprint requests to John J. Fernandes, DO, professor of pathology, Department of Pathology, Olympia Fields Osteopathic Hospital and Medical Center, 20201 Crawford Ave, Olympia Fields, IL 60461. rently reported -not reference or referent values-identifies a range of values representing a statistical distribution from the mean, encompassing $95 \%$ of the values of the test population. This distribution forms the standard bell curve with calculated deviations from the mean (Fig 1). These Gaussian statistics are based on Gauss' law of errors, and applies only to repeated measurements on the same subject, not to a series of measurements on different subjects. These results tell us nothing about morbidity or mortality, and they are unrelated to the prevalence of a disease. Gauss' law of errors implies that a plot measurement made on the same individual would fit a normal distribution, a Gaussian distribution. This implication does not take into account health or disease, and reports to clinicians only values that are only most commonly encountered in various groups. Clinicians dealing with one individual patient are not concerned with frequency distribution in populations, but with individual patients and their diagnostic needs.

When "normal range" is defined as the central $95 \%$ of a series of measurements from a group of healthy people, this definition means that $5 \%$ of these healthy people are called "abnormal." In addition, it also means that if we are healthy, we have a $5 \%$ chance of being labeled "abnormal." The more testing done on a patient, the more likely it is that the results of at least one test will be abnormal (Table 1). As we increase the number of tests, the likelihood of the patient's being labeled "abnormal" increases proportionately. ${ }^{1}$ Thus, in chemical profiling or screening, when a healthy person undergoes 20 independent tests, the like- 


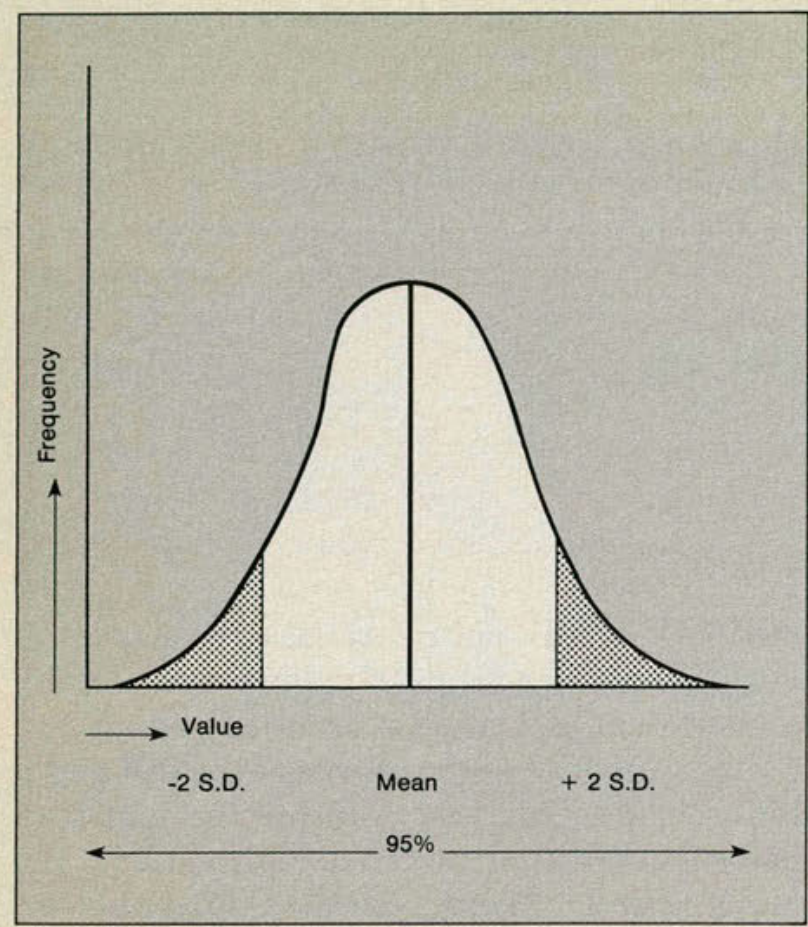

Figure 1. Standard bell curve with calculated deviations from the mean. This represents the normal range for most laboratory tests, with results defined as falling within \pm 2 standard deviations (SD) from the mean (95\%) of all results.

lihood of that person's being labeled "abnormal" is greater than $50 \%$. But in addition, when 20 tests are done as a screen, as on a routine hospital admission screen, there is only a $36 \%$ chance that the results of all 20 tests will be normal.

Routine screening and routine hospital admission laboratory testing have created a situation in which, based on chance alone (Gaussian distribution), some results would be reported as "abnormal" in an otherwise healthy patient. In published studies, these abnormal test results that do not reflect disease have been calculated to be as high as a third of biochemical screening. ${ }^{2}$

\section{Sensitivity and specificity}

The sensitivity of a laboratory test is the incidence of true-positive results obtained when the tests apply to a group known to have the disease. It is the probability that a test result will be abnormal, true-positive in disease. Specificity of a laboratory test is the incidence of true-negative results when the test is applied to a group known to be free of the disease. It is the probability that a test result is normal in the absence of disease. Sensitivity is equal in percentage to positive results obtained in subjects with disease, and specificity is the percentage of normal results obtained in healthy subjects.

Because some overlap always exists between health and disease, it follows that no test is $100 \%$ specific or $100 \%$ sensitive (Fig 2). The cutoff point, or upper limit of normal value chosen for a laboratory test to distinguish between health and disease, defines sensitivity and specificity. If the cutoff point is placed at $A$, everyone with disease is termed "abnormal," and sensitivity is $100 \%$ (Fig 3). However, in this circumstance, given normal overlap between health and disease, a significant segment of the healthy group is termed "abnormal" (false-positive results).

If the cutoff point is placed at $B$, everyone without disease is termed "normal," and specificity is $100 \%$ (Fig 4). Again, given normal overlap between health and disease, a substantial segment of the patients with disease will have normal (false-negative) results. When the cutoff point is moved, sensitivity and specificity become reciprocal; that is, as sensitivity is increased, specificity decreases, and vice versa. When a single limit is used, either absolute

\begin{tabular}{|cc|}
\hline $\begin{array}{c}\text { Table 1 } \\
\text { Chance of Abnormal Result in Relation } \\
\text { to Number of Tests a Patient Has }\end{array}$ \\
\hline $\begin{array}{c}\text { No. of } \\
\text { independent } \\
\text { tests }\end{array}$ & $\begin{array}{c}\text { Probability } \\
\text { of abnormal } \\
\text { result, } \%\end{array}$ \\
\hline 1 & 5 \\
2 & 10 \\
5 & 23 \\
10 & 40 \\
20 & 64 \\
50 & 92 \\
\hline
\end{tabular}




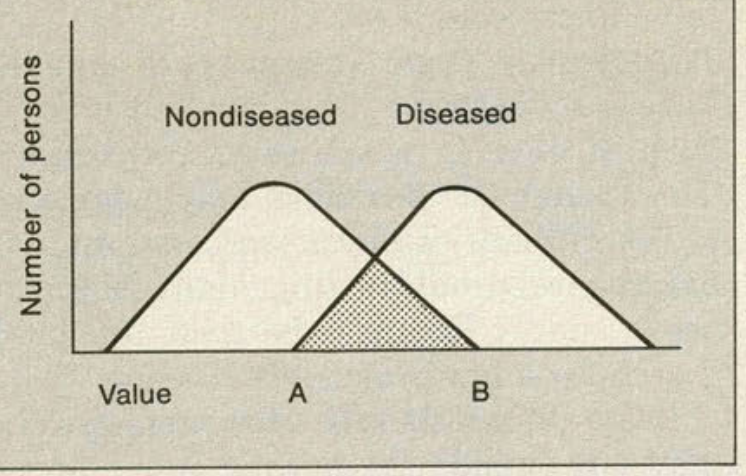

Figure 2. The overlap that exists between health and disease in laboratory results is expressed as a value between points A and B. Patients falling in this zone may have their condition misdiagnosed.

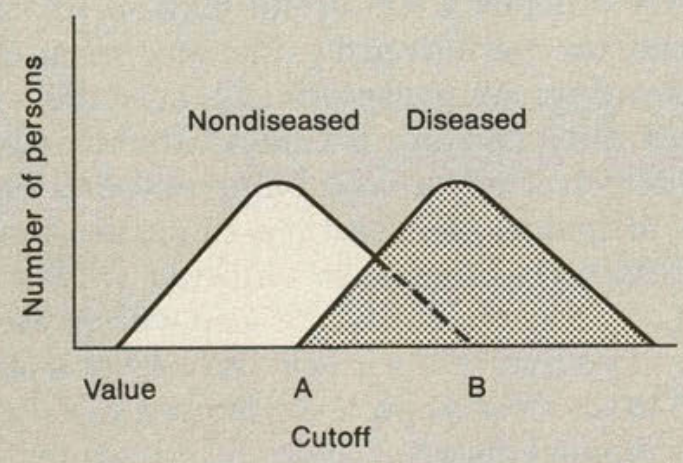

Figure 3. With cutoff point placed at A, everyone with disease is termed "abnormal"; sensitivity is $100 \%$. However, in normal overlap, a substantial segment of the group without disease falls in "abnormal" range.

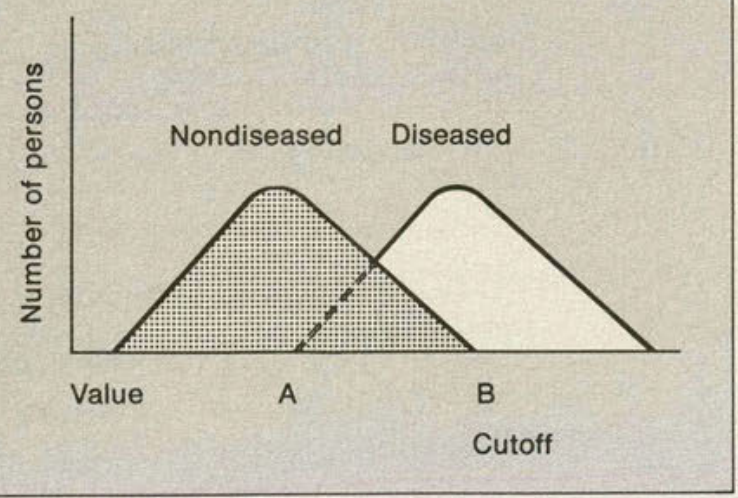

Figure 4. With the cutoff point placed at B, everyone without disease is termed "normal"; and specificity is $100 \%$. sensitivity or absolute specificity can be achieved, never both.

Sensitivity and specificity can be altered by combination testing. ${ }^{3}$ Using three hypothetical tests, $A, B$, and $C$ with listed sensitivity and specificity, we can increase specificity and decrease sensitivity with serial testing $(A, B$, and $C$ ), and increase sensitivity and decrease specificity with parallel testing $(A, B$, or $C)$. (See Table 2.) Sick patients are more likely to have one of three results abnormal than to have all three abnormal and, therefore, sensitivity increases in parallel testing. Also, a patient without disease is more likely to have a single abnormal laboratory test than multiple abnormalities; therefore, specificity increases with serial testing. Parallel testing decreases false-negative results, and serial testing decreases false-positive results.

\section{Prevalence and predictive value}

Prevalence is the likelihood of disease being present before any testing is done. When expressed as a rate, prevalence equals the number of patients per 100,000 who have disease at the time of analysis. The incidence rate, however, expresses the number of patients per 100,000 in whom the disease develops in a 1 year period. The predictive value of a positive test is the percentage of positive results that are true-positive when a test is applied to a population containing both healthy and ill subjects; it is the probability that a positive test will be correct.

The relationship of the properties of sensitivity and specificity with predictive value and prevalence are expanded in Table 3 . As the prevalence of a disease increases, with sensitivity and specificity held constant, the predictive value of a test increases (Fig 5). Also, false-negative rates increase, and false-positive rates decrease. ${ }^{3}$ The combination of these effects can be seen in Table 4 . Here, a test having $90 \%$ sensitivity and $90 \%$ specificity is used to detect disease in two different circumstances in adults.

In the first circumstance, there is a high prevalence rate of $50 \%$, as in coronary artery disease; and, in the second example, the prevalence rate is $1 \%$, as in some infrequently oc- 


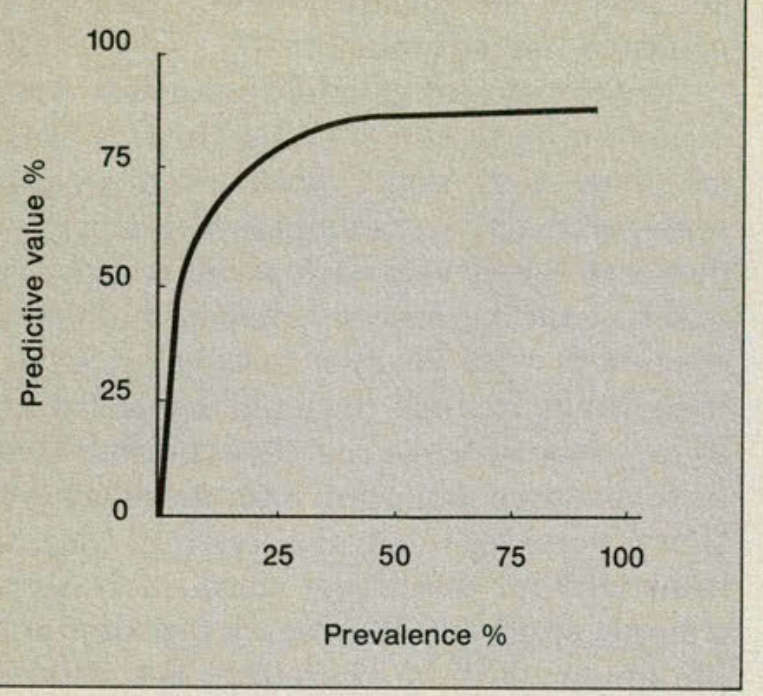

Figure 5. Predictive value of test increases as prevalence of a disease increases, with sensitivity and specificity held constant.

curring primary malignancies such as primary hepatoma. If 200 people are tested and the prevalence is $50 \%, 100$ of them are healthy and 100 have the disease. Because the test is $90 \%$ sensitive and $90 \%$ specific, there will be 90 true-positive, 10 false-negative, 90 truenegative, and 10 false-positive results. Therefore, 100 positive results and 100 negative results are obtained. Of the 100 positive results, 90 are true-positive; thus, the likelihood that a positive result is truly positive is $90 \%$. This percentage would represent the predictive value of a positive test. Therefore, when a physician orders this test in the clinical environment, where the likelihood of the disease is $50 \%$, a positive result means that there is a $90 \%$ chance the disease is present.

When the prevalence is $1 \%$, as in the second example, the results are quite different. If 10,000 people are tested and the prevalence is $1 \%, 100$ have the disease, and 9,900 do not, there will be 90 positive, 10 false-negative, 8,910 true-negative, and 990 false-positive results. The likelihood that any positive result is truly positive is approximately $8 \%$. Therefore, a positive result in this circumstance would more than likely suggest health rather than disease. In this circumstance, when disease is suspected clinically, positive results are likely to be positive; however, in asymptoma- tic patients, positive results are more likely to be false-positive.

Contrarily, negative results are more likely to be truly negative in asymptomatic patients than in those in whom disease is suspected. This likelihood, over time, may explain some of the difficulties physicians encounter with biochemical profile testing. Many false results occur simply because the tests are used to search for a low-prevalence disease.

Laboratory tests with a low predictive value may fall outside the normal range and provide little clinical information, although they often show up on panels. The serum chloride and the lactic dehydrogenase $(\mathrm{LDH})$ assays are examples of this phenomenon. Electrolyte panels routinely include chloride and, although chloride is a major cellular anion, the chloride assay provides little useful clinical information in the routine setting. Elevated serum chloride values are nonspecific, because they may represent a diversity of clinical situations, such as dehydration, renal tubular acidosis, diabetes insipidus, and reactions to medications.

Similarly, decreased chloride values are equally nonspecific and are seen with processes that lose hydrochloric acid. Ravel, ${ }^{4}$ in a study of 649 consecutive patients, found a significant discrepancy between chloride and sodium, and only $1.5 \%$ of cases. Hyperchloremia no more implies chloride excess than hyperneutremia implies sodium excess; and hypochloremia, like hyponeutremia, does not reflect the state

\begin{tabular}{|lcc|}
\hline \multicolumn{3}{|c|}{$\begin{array}{c}\text { Table 2 } \\
\text { Sensitivity and Specificity } \\
\text { of Combination Testing }\end{array}$} \\
\hline Test(s) & Sensitivity, \% & Specificity, \% \\
\hline A & 90 & 90 \\
B & 92 & 93 \\
C & 95 & 95 \\
$\begin{array}{c}\text { Serial testing } \\
\text { (A + B + C) }\end{array}$ & 75 & 100 \\
$\begin{array}{c}\text { Parallel testing } \\
\text { (A or B or C) }\end{array}$ & 100 & 75 \\
\hline
\end{tabular}




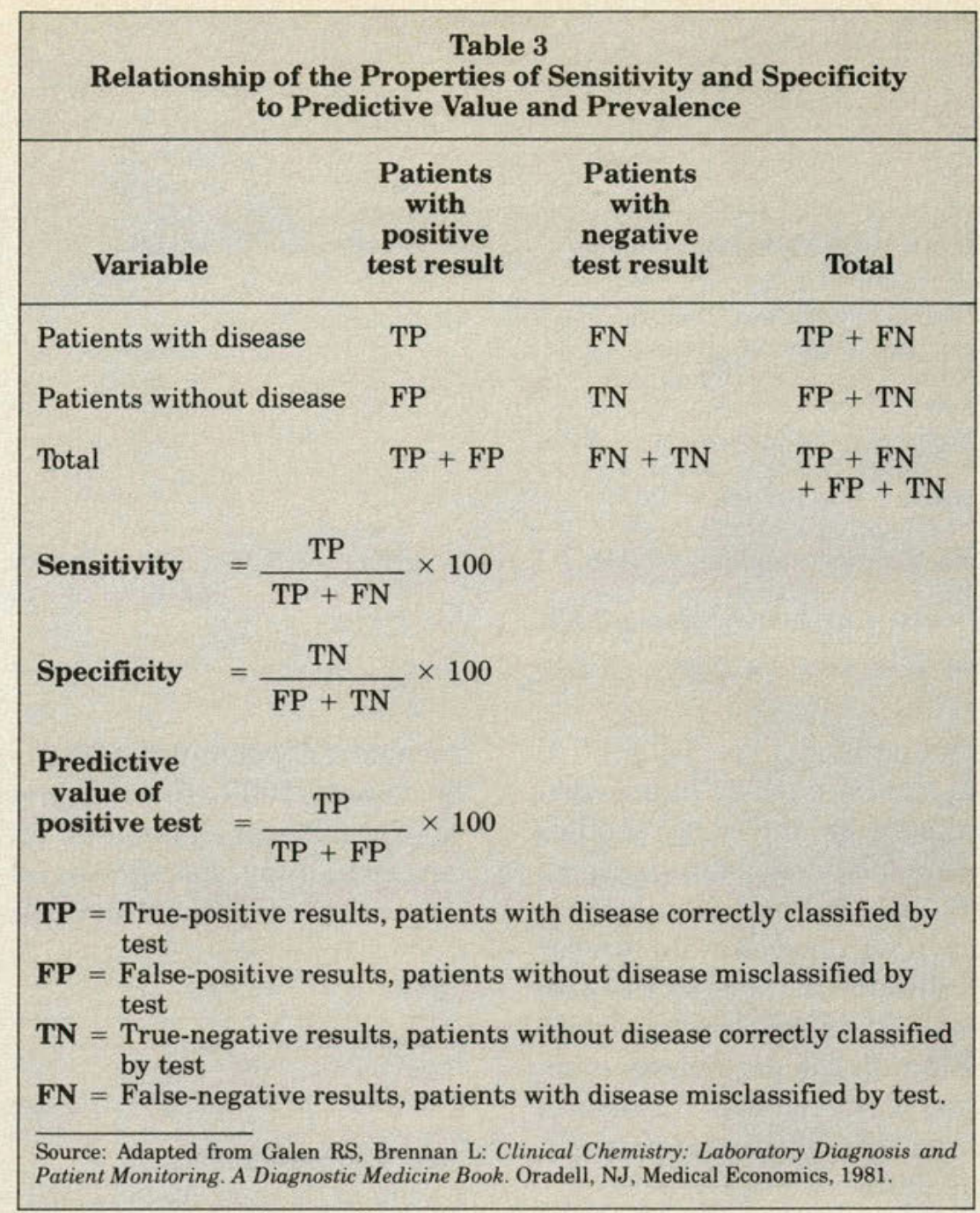

of iron depletion. ${ }^{5}$ The positive predictive value of serum chloride is low.

An abnormal LDH level in the absence of a known clinical process or other laboratory abnormality, is of little diagnostic value. Elevated $\mathrm{LDH}$ levels are seen in neoplastic disease, hypoxia, hemolytic anemias, myocardial infarction, liver dysfunction, and in general categories like pulmonary and renal diseases. The presence of elevated LDH values more frequently creates confusion because of the assay's nonspecificity. In a study at the Cleveland Clinic, ${ }^{6}$ screening tests of 2006 patients revealed 22 elevations of $\mathrm{LDH}$. Further analysis with isoenzymes revealed three of the elevations to be due to cardiac ischemia, liver dysfunction, or hemolysis; 13 elevations were nondiagnostic, and the remainder were considered to be falsely positive. How often, in the pursuit of clinical excellence, do these falsely abnormal findings contribute to the cost of medical care?

The erythrocyte sedimentation rate (ESR), as a screening test in an asymptomatic population, will be the only clue to serious illness in $0.06 \%$ of patients. ${ }^{7}$ Even in elderly nursing home residents with multisystem disease, the predictive value of a moderately elevated ESR is less that $12 \% .^{8}$ However, if the ESR is greater than $100 \mathrm{~mm} / \mathrm{h}$, the predictive value increases. Although the predictive value of this test for any given disease is low, when all patients with infection, malignancy, renal disease, and inflammatory disease are taken together, the predictive value of the ESR greater than $100 \mathrm{~mm} / \mathrm{h}$ is about $90 \% .^{9}$ 
Table 4

Effects of Disease Prevalence on Positive Predictive Value

\begin{tabular}{|c|c|c|c|}
\hline Variable & $\begin{array}{c}\text { No. with } \\
\text { positive } \\
\text { test } \\
\text { result }\end{array}$ & $\begin{array}{c}\text { No. with } \\
\text { negative } \\
\text { test } \\
\text { result }\end{array}$ & Total \\
\hline \multicolumn{4}{|l|}{ Disease prevalence $=\mathbf{5 0} \%$} \\
\hline No. of patients with disease & 90 & 10 & 100 \\
\hline No. of patients without disease & 10 & 90 & 100 \\
\hline Total & 100 & 100 & 200 \\
\hline \multicolumn{4}{|l|}{ Positive predictive value $=90 \%$} \\
\hline \multicolumn{4}{|l|}{ Disease prevalence $=1 \%$} \\
\hline No. of patients with disease & 90 & 10 & 100 \\
\hline No. of patients without disease & 990 & 8,910 & 9,900 \\
\hline Total & 1,080 & 8,920 & 10,000 \\
\hline Positive predictive value $=8.3 \%$ & & & \\
\hline
\end{tabular}

The predictive value model can be applied to other areas of medicine, such as interpretation of electrocardiograms and x-ray studies as well as history and physical examinations. A thorough history and physical examination of a given population will increase the prevalence of a disease, thereby increasing the predictive value of any test or procedure used on that population. Finally, the predictive value model, when applied to the medical literature, can enhance the reader's ability to properly interpret scientific data.

\section{Comments}

The relative importance of sensitivity, specificity, predictive value, and prevalence of disease for profiling and for exclusion or confirmation of an hypothesis is described. High sensitivity is a major profiling requirement. High specificity and positive predictive value should be sufficient to reduce the number of falsepositive results to a reasonable level. As a general rule, high sensitivity is a major requirement for definite exclusion, and high specificity and positive predictive value are major necessities for definite confirmation. When it becomes necessary in clinical decision-making to exclude a dangerous disease, sensitivity should be close to $100 \%$. Similarly, when it is necessary to confirm a diagnosis that requires dangerous therapy, specificity and positive predictive value are crucial indicators.

1. Murphy EA: The Logic of Medicine. Baltimore, Md, Johns Hopkins Press, 1976.

2. Bradwell AR, Carmalt MHB, Whitehead TP: Explaining the unexpected abnormal results of biochemical profile investigations. Lancet 1974;2:1071.

3. Galen RS, Gambino SR: Beyond Normality: The Predictive Value and Efficiency of Medical Diagnosis. New York, NY, John Wiley \& Sons, Inc, 1975.

4. Ravel R: Clinical Laboratory Medicine: Clinical Applications of Laboratory Data, ed 5. Chicago, Ill, Year Book Medical Publishers, 1989.

5. Hall WD: Serum chloride, in Walker HK, Hall WD, Hurst JW (eds): Clinical Methods: The History, Physical and Laboratory Examinations. Stonebarn, Mass, Butterworth, 1976.

6. Van Lente F, Castellani W, Chou D, et al: Application of the expert consultation system to accelerated laboratory testing and interpretation. Clin Chem 1986;32:1719.

7. Sox HC Jr, Liang MH: The erythrocyte sedimentation rate: Guidelines for rational use. Ann Intern Med 1986;104:515.

8. Tinetti ME, Schmidt A, Baum J: Use of the erythrocyte sedimentation rate in chronically ill elderly patients with a decline in health status. Ann J Med 1986;80:844.

9. Fischer RM, Page MI: Clinical significance of extreme elevation of the erythrocyte sedimentation rate. Arch Intern Med $1986 ; 146: 1581$ 


\title{
Coming soon from BASEL Pharmaceuticals Division of CIBA-GEIGY Corporation
}

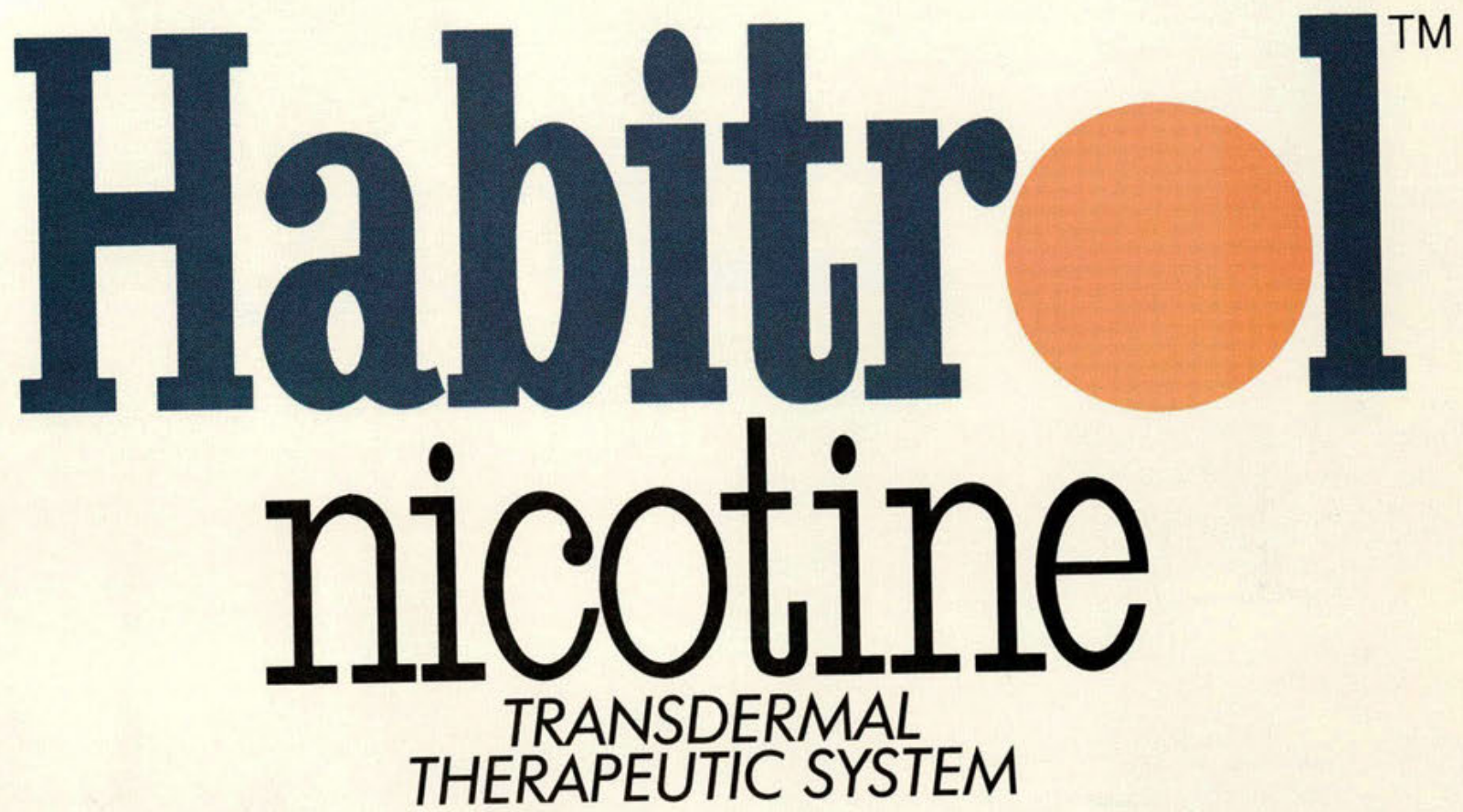

\author{
๑BASEL \\ V. Pharmaceuticals \\ Division of CIBA-GEIGY Corporation
}




\section{IN LOWER \\ RESPIRATORY INFDCTIONS}

Your patients deserve first-tinze success*..

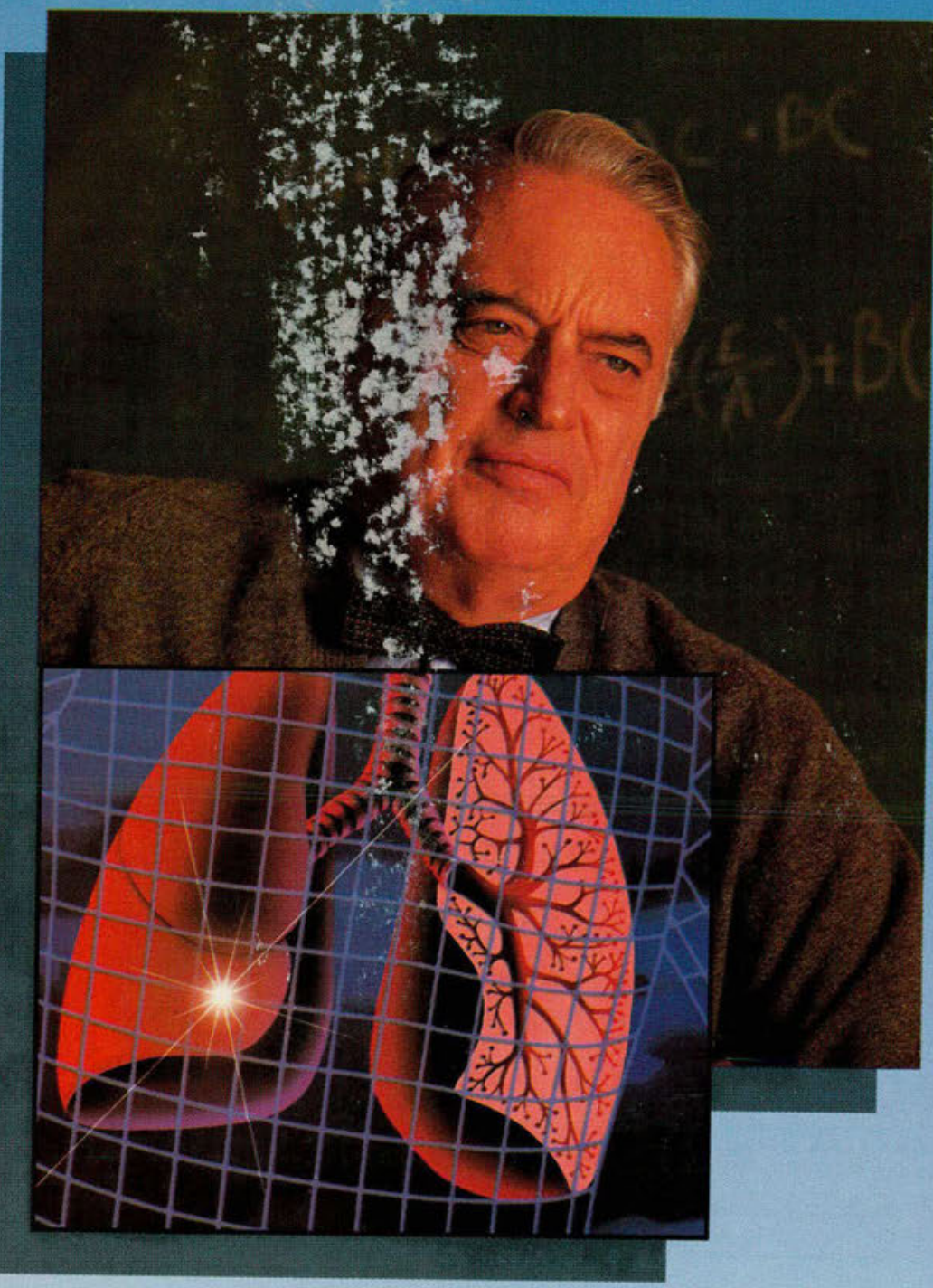

\section{Prescribe
amoxicllin/clarulanate potassium}

First-line

for all the right reasons
${ }^{*}$ Clinical success rate for bronchitis and pneumonia was $98 \%$. Data on file, Medical Department, SmithKline Beecham Pharmaceuticals. C. SmithKline Beecham, 199

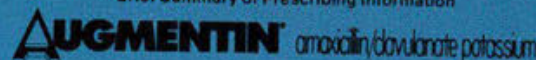

Indications and Usage: A Avomentro is indicated in the treatment of intectin

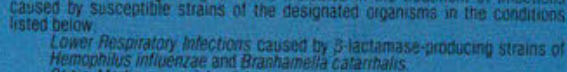

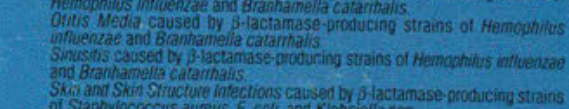

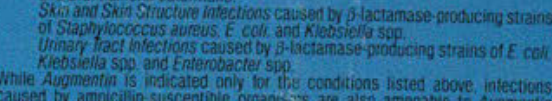

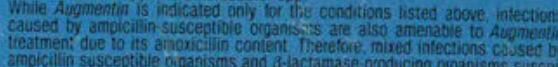

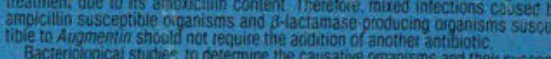

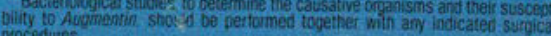
Therapy may be instrified prior to obtaining the results trem bacteriologic

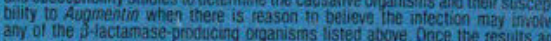
known therapy shousio be zglusted, if aporcopriate. WAANANGS:

PE ALS WTHA A<smiles>[GeH2]=[W]</smiles>

AN

REACTIONS REOUIRE IMMEDIATE FMERGGNEY TRSATS ANAPHYLACTOI RINE. OXYGEN INTRAVENOUS SIEROIDS. AND AIRWAY MANAGGEMENT IN PLIDDING INTUBATION. SHOULD ALSO BE ADMINISTERED AS INDICATEO.

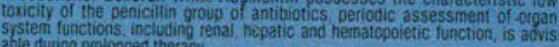

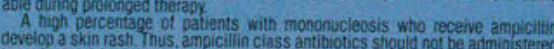

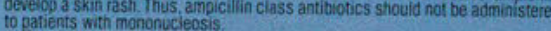
The possibility of superintections with mycotic or bacterial pathogens shoult Pseudomonas of Candita, the drop should ve discontinued and/ar appropiriate Orug literactions: Probenecid decreases the renat tubular secretion of amoricitin
Concurrent use with Augmentin may sesuth in increased and orolonged bloor Conculs of amoxicilitin Augmentin may result in increased and prolonged bloo tialily concurrent administration of allopurinol and ampicilinin increases substanpattients receeving ampicilin alone it is not knows whether (his potentiation of

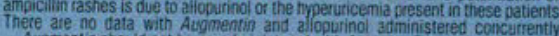
Carcinogenesis, Mutagenesis, Im Pregnancy Category B: Pegroduction studies have been performed in mice and of $\mathrm{imp}$ aises

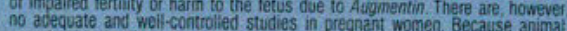
reprocuction studies are not always predictive of human response, this drug

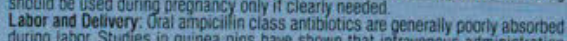

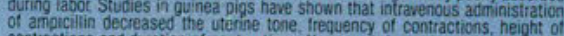
cantuactions and duration of contactions, However it is not known whether the

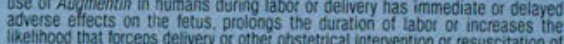
Nursing Mothers. Amoicsilin class antibiotics are exceted in the milk theretore Adverse Reactions: Augmentin is generally weil tolerated The majority of side effects observed in cinicaif trais were of a mild and transient naturearad less

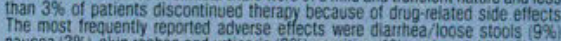

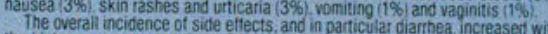

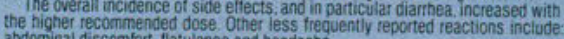
The following adverse reactions have been reported tor ampicillin clas Gastronitestinat Diarnhea, nausea voniting indigestion, gastritis stomaztitis. gosssitis,

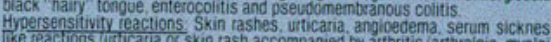
and trequenty fever), erythema multiforme irately Stevens.Johinson Syndorome and an occasional case of extoliative dermatitis have been reported Thes reactions may je controlled with antihistamines and if necessaryy systemi unless the opinion of the physician dictates otherwise Serious and occasion Warnings. Liver A moderate rise in SGOT SGPT AST, and/or ALT has been noted in patients

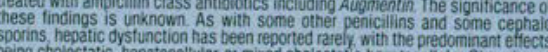

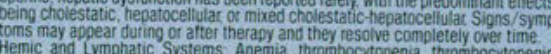
Hemic and Lymphatic Systems. Anemia, thrombocytopenia, thrombocytopen

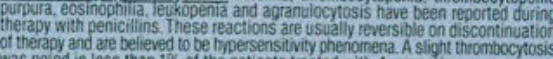
was noied in less than $1 \%$ of the patients treated with Augmentin

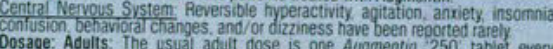

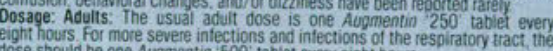
Since both the Augmentin "250" and '500" tablets contain the same amount are not equivalent to one Augmentin '500 $250^{\prime}$ tablets should not be substituted for one Augmentifin' '500' tablet tor Chilitren: The usual dose is $20 \mathrm{mg} / \mathrm{kg} /$ day, based on amoxiciltin component, in

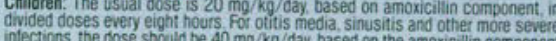
in divided doses every eight hours. Aliso avaliable as Aupumentin 125 and

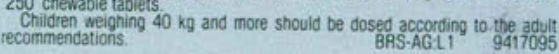

\section{SB}

SmithKIIne Beecham

Pharmaceuticals

Philadelphia, PA 19101 